\title{
Virologic testing in bronchiolitis: does it change management decisions and predict outcomes?
}

\author{
Fabiola Stollar • Gabriel Alcoba • Alain Gervaix • \\ Constance Barazzone Argiroffo
}

Received: 5 December 2013 /Revised: 1 May 2014 / Accepted: 2 May 2014 / Published online: 25 May 2014

(C) Springer-Verlag Berlin Heidelberg 2014

\begin{abstract}
The aim of this study was to evaluate the clinical, therapeutic, laboratory, and radiological differences between respiratory syncytial virus (RSV) and non-RSV bronchiolitis in order to assess if the prior knowledge of viral etiology changed management decisions and would be able to predict outcomes. Medical charts of children $<1$ year admitted to the emergency department with bronchiolitis during two RSV seasons (2010-2012) were reviewed. We analyzed 221 episodes of bronchiolitis. The percentage of exams performed (95\% confidence interval (CI) 0.74-2.52), abnormal laboratory and radiological findings (95\% CI 0.53-16.89) did not differ between groups. RSV bronchiolitis had a more severe clinical course. However, virologic testing for RSV had low specificity in identifying at-risk patients for hospitalization, longer hospital length of stay, and need of oxygen therapy and nasogastric tube (44, 40, 42, and $35 \%$, respectively), and while statistically significant, the positive likelihood ratios were only slightly greater than 1. Conclusion: Although RSV bronchiolitis has a more severe clinical course, virologic testing does not help in management decisions, and at an
\end{abstract}

Communicated by David Nadal

F. Stollar $(\bowtie) \cdot$ C. B. Argiroffo

Pediatric Pulmonology Unit, Children's Hospital, University

Hospitals of Geneva, Rue Willy-Donzé 6, 1211 Geneva 14,

Switzerland

e-mail: fabipediatria@hotmail.com

C. B. Argiroffo

e-mail: constance.barazzone@hcuge.ch

G. Alcoba $\cdot$ A. Gervaix

Pediatric Emergency Division, Children's Hospital, University

Hospitals of Geneva, Geneva, Switzerland

G. Alcoba

e-mail: gabriel.alcoba@hcuge.ch

A. Gervaix

e-mail: alain.gervaix@hcuge.ch individual level, as a performance test, it seems insufficient to precisely predict outcomes.

Keywords Bronchiolitis · Disease severity · Outcomes · Respiratory syncytial virus · Virologic testing

$\begin{array}{ll}\text { Abbreviations } \\ \text { CBC } & \text { Complete blood count } \\ \text { CI } & \text { Confidence interval } \\ \text { CRP } & \text { C-reactive protein } \\ \text { ED } & \text { Emergency department } \\ \text { hMPV } & \text { Human metapneumovirus } \\ \text { ICD } & \text { International Classification of Diseases } \\ \text { IQR } & \text { Interquartile range } \\ \text { ICU } & \text { Intensive care unit } \\ \text { LOS } & \text { Hospital length of stay } \\ \text { LR } & \text { Likelihood ratio } \\ \text { NGT } & \text { Nasogastric tube } \\ \text { OR } & \text { Odds ratio } \\ \text { PCR } & \text { Polymerase chain reaction } \\ \text { RSV } & \text { Respiratory syncytial virus } \\ \text { SD } & \text { Standard deviation } \\ \text { SpO } & \text { Pulse oxygen saturation } \\ \text { WBC } & \text { White blood cell }\end{array}$

\section{Introduction}

Bronchiolitis is the most frequent cause of pediatric hospital admissions during the winter months in early childhood, with respiratory syncytial virus (RSV) being the causative organism in most cases; the proportion of RSV and non-RSV bronchiolitis changes from year to year [1]. It is a major problem of public health, causing 3.4 million hospitalizations and between 66,000 and 199,000 deaths annually, globally, at 
an estimated annual cost of more than $\$ 700$ million per year $[1,12]$.

The clinical course of a child with bronchiolitis is variable, and the potential for disease progression has led to research to identify risk factors whose presence may indicate a severe form of disease or may be predictive of complications and lifethreatening, thus help physicians in the emergency department (ED) $[13,14]$.

Research on prediction of the clinical course of bronchiolitis has revealed comorbidities and young age as important risk factors for developing severe disease and specific physical findings as less predictive of outcomes for the majority of cases $[1,5,16]$. During the last decade, a better understanding of bronchiolitis etiology and the emergence of more advanced techniques for viral detection have raised the question of the relevance of the etiologic agent in the course of the disease $[8,15]$.

Several studies have investigated whether the infection etiology contributes to the bronchiolitis severity and have advocated that the RSV episodes are more severe, in contrast to some other viruses as the human metapneumovirus (hMPV) which affects older infants and probably has a milder course [2, 6-8]. However, the majority of these studies only compared the severity of different etiologic groups but did not assess whether the prior knowledge gained from such testing could change management decisions or predict outcomes. Moreover, bronchiolitis guidelines advocate that the clinical relevance of identifying the specific etiology of a child with severe disease is still uncertain [18].

In this study, we evaluated the clinical, therapeutic, laboratory, and radiological differences between RSV and non-RSV bronchiolitis in order to assess if the prior knowledge of the viral etiology in the ED led to changes in the routine management and would be able to predict outcomes in these patients.

\section{Materials and methods}

\section{Study design and subjects}

This was a retrospective, observational study during two consecutive RSV seasons (2010-2012). We included infants ( $<1$ year of age) admitted to the ED of the University Hospital of Geneva with a diagnosis of acute bronchiolitis. Patients with chronic diseases such as congenital heart disease, bronchopulmonary dysplasia, trisomy 21 , congenital or acquired immunodeficiencies, and neuromuscular disorders or without a viral test (detection of RSV) were excluded. Episodes of acute bronchiolitis were identified via International Classification of Diseases (ICD), version 2010, with the following discharge codes: acute bronchiolitis (J21), RSV bronchiolitis (J21.0), and acute unspecified bronchiolitis
(J21.9). After reviewing the medical records, only children who had the typical clinical presentation with rhinitis, tachypnea, wheezing, cough, crackles, use of accessory muscles, and/or nasal flaring were included [1]. This study was approved by the institution's ethical committee on clinical research in children (protocol 12-026).

Data collection

Variables of interest were identified from previous studies [16], and medical charts were reviewed for the following data: demographic and epidemiologic (age, gender, gestational age, and date of admission); underlying medical conditions; bacterial coinfections (sepsis, acute otitis media, pneumonia, and urinary tract infection); radiological (chest X-ray) and laboratory tests (detection of RSV, C-reactive protein (CRP), complete blood count $(\mathrm{CBC})$, and capillary blood gas analysis $\left(\mathrm{pCO}_{2}\right.$ and $\left.\mathrm{pH}\right)$ ); medications received (inhaled $\beta$-receptor agonist, systemic steroids, and antibiotics); and disease severity parameters (mortality, hospitalization, hospital length of stay (LOS), intensive care unit (ICU) admission, requirement and duration of nasogastric tube (NGT), supplemental oxygen, and mechanical ventilation).

Regarding the laboratory and radiologic data, only tests including CBC and CRP, capillary blood gas analysis, and chest X-ray performed during the first 12,6 , and $24 \mathrm{~h}$ of admission, respectively, were included. For blood analysis, we considered abnormal laboratory values as $\mathrm{pCO}_{2}>6 \mathrm{kPa}$, $\mathrm{pH}<7.35$ or $>7.45, \mathrm{CRP}>10 \mathrm{mg} / \mathrm{L}$, and $\mathrm{WBC}<4.5$ or $>10 \mathrm{~g} / \mathrm{L}$.

Respiratory samples were collected by nasopharyngeal aspiration during the first hour in the ED and then stored; normally, virologic testing was performed for all hospitalized patients during the RSV season and otherwise in some cases for children at risk of hospitalization. The viral diagnostic tests used included the QuickVue ${ }^{\circledR}$ RSV test (enzyme-linked immunoassay) and/or individual one-step real-time Taqman $(-$-based polymerase chain reaction (PCR) or reverse transcription PCR, as routinely performed in our virology laboratory.

To precisely calculate the LOS, we used the ED triage time and the hospital discharge order time. Clinical data at presentation to the ED were noted, including the duration of symptoms, respiratory rate, presence of wheeze, clinical assessment of degree of retractions (assembled for analysis into none/mild versus moderate/severe), pulse oxygen saturation $\left(\mathrm{SpO}_{2}\right)$, and the presence of feeding difficulties, cyanosis, and apnea.

Standards of care

Criteria of hospitalization used in our hospital were those published by Barben et al. [3]: children feeding less than 
$50 \%$ of the required amount, $\mathrm{SpO}_{2}$ of $<92 \%$ in air, progressive respiratory failure, apnea, bradycardia, and poor social conditions.

Infants were nourished via NGT when intake was below $50 \%$ of normal or when excessive work of breathing was noted. Supplemental oxygen was administered (through nasal cannulae) for $\mathrm{SpO}_{2}$ of $<92 \%$ in air. Following recommendations of bronchiolitis guidelines [1], sometimes especially in patients older than 6 months, a trial of inhaled $\beta$-receptor agonist treatment was attempted in the ED. The treatment was continued only after a proven benefit such as increase in $\mathrm{SpO}_{2}$ and decrease of respiratory rate and wheezing. Oral steroids were not routinely used, and finally, antibiotics were administered when concomitant bacterial infection was suspected. Oxygen therapy was stopped once values for $\mathrm{SpO}_{2}$ in air were $\geq 92 \% . \mathrm{SpO}_{2}$ monitoring was continuous (Philips model M3 oximeter), with a 10-h observation period after removal before discharge.

\section{Statistics}

Results are presented as mean \pm standard deviation (SD) or median with interquartile range (IQR) for continuous variables and as percentages for categorical variables. Continuous variables were analyzed using Student's $t$ test or Mann-Whitney test and categorical variables by the $\chi^{2}$ test or Fischer's exact test, as appropriate, and the $95 \%$ confidence interval (CI) for odds ratio (OR) was determined according to standard methods. A $P$ value $<0.05$ was considered statistically significant. All the analyses were performed by using Stata 12.0 (StataCorp, College Station, TX, USA).

To evaluate the differences in clinical course and disease severity between children with RSV and non-RSV bronchiolitis, we compared rates of hospitalization, longer LOS ( $>3$ days; defined using the median value of 3 days), supplemental oxygen (including invasive and noninvasive ventilation) and NGT requirements, longer duration of oxygen therapy ( $>2$ days; defined using the median value of 2 days), longer duration of NGT ( $>3$ days; defined using the median value of 3 days), ICU admission, reconsultation after discharge, and mortality.

Diagnostic accuracy statistics including sensitivity, specificity, and positive and negative likelihood ratios (LR+ and LR-) were calculated for variables of disease severity that were statistically different between the RSV and non-RSV groups.

We retrospectively calculated the power and for the first outcomes (hospital length of stay, need of oxygen therapy and NGT), using the sample size of 221 episodes, gave 85.7$99.6 \%$ power to detect differences between the two groups, with a 0.05 two-sided significance level.

\section{Results}

Study group

There were 479 infants (582 episodes) under 1 year and without chronic diseases presenting to the ED with bronchiolitis. In contrast to the literature [18], we had 184/582 (32\%) of hospitalizations considering all the patients with the diagnosis of bronchiolitis visiting the ED in the study period. In our study, we included 202 patients (123 M, 79 F) and 221 episodes of bronchiolitis in which viral diagnostic tests were performed, 157 being RSV positive and 64 negative (nonRSV). There was a mean of $1.1 \pm 0.34$ episodes per patient (range, 1-3). The mean age was $4.57 \pm 3.4$ months (range, 0.3 11.8). Patients with RSV bronchiolitis were younger than nonRSV patients $(4.2 \pm 3.4$ vs. $5.4 \pm 3.3$ months, $P=0.0231)$. Figure 1 shows the monthly distribution of episodes of bronchiolitis during the seasons 2010/2012.

Emergency department presentation

ED presentation among the groups is shown in Table 1. The groups did not differ with respect to the initial clinical presentation. The frequency of comorbidities such as pneumonia, sepsis, acute otitis media, and urinary tract infection also did not differ between the groups.

\section{Medications received}

The medications received are summarized in Table 2. The medications used during the week leading up to the ED visit and the medications received at discharge did not differ between the groups. As opposed to medical recommendations against the excessive use of a bronchodilator in bronchiolitis treatment [18], we found a high percentage of patients who received inhaled $\beta$-receptor agonist treatment in both groups. Patients with RSV episodes were less likely to receive inhaled $\beta$-receptor agonist treatment at the hospital (95\% CI 0.24 $0.80)$. We also analyzed the patients who had received inhaled $\beta$-receptor agonist treatment before arriving at the ED and if the treatment was stopped in the hospital; we found no statistical difference between RSV and non-RSV groups (95\% CI 0.17-1.72).

Laboratory tests and radiological findings

The laboratory or radiological exams were performed in 84 (38\%) episodes, and the number of exams did not differ between the groups (95\% CI 0.74-2.52). Among children who had capillary blood analysis (blood gas (57 samples), CBC (35 samples), and CRP (35 samples)), the groups did not differ with respect to abnormal levels of $\mathrm{pCO}_{2}$ (95\% CI 0.9 23.14) and pH (95\% CI 0.22-4.26), white blood cell (WBC) 


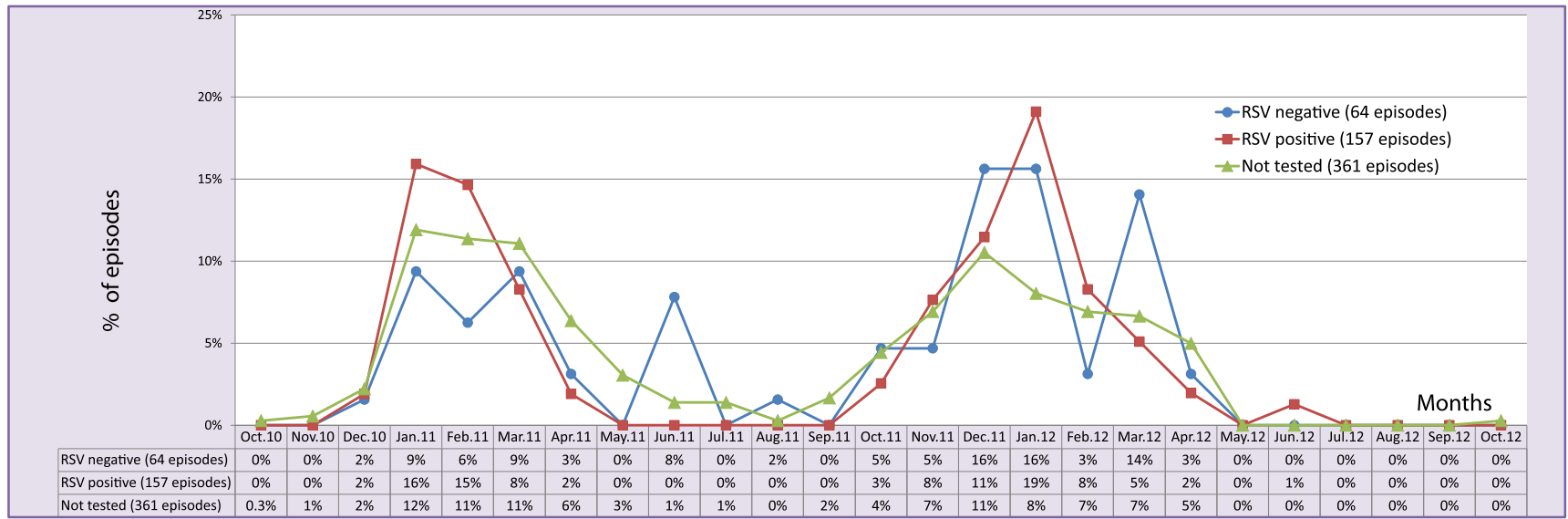

Fig. 1 Monthly distribution of bronchiolitis episodes during the study period (2010-2012)

count (95\% CI 0.05-1.82), and CRP (95\% 0.41-10.52). A chest radiograph was performed in 54 (24\%) episodes, and the radiological pattern most commonly described for both groups was the presence of bronchial wall thickening/ hyperinflation followed by focal opacity and atelectasis. The two groups also did not differ with respect to the percentage of abnormal chest radiographic findings (95 \% CI 0.53-16.89).

Differences in clinical course and disease severity between RSV and non-RSV bronchiolitis

The median LOS, duration of oxygen therapy, and duration of NGT for all episodes were 3 days (IQR 1.5-5.1), 2 days (IQR 1.2-3.8), and 3 days (IQR 1.7-4.5), respectively. Compared to the non-RSV group, the RSV group had higher rates of hospitalization and longer LOS and duration of oxygen therapy. The number of children who required supplemental oxygen and NGT was also higher for children with RSV bronchiolitis (Table 3 ). These results remained significant even when the OR was adjusted for age.

To avoid bias related to our criteria of hospitalization, we analyzed the rates of oxygen therapy and NGT only in hospitalized children; the higher rates of oxygen therapy in the RSV group remained statistically significant (86 vs. $67 \%$, $P=0.007$ ), and there was also a nearly significant trend toward higher rates of NGT (51 vs. $35 \%, P=0.06$ ) in this group. We documented no deaths.

Subanalysis in patients under 3 and 6 months

We performed a subanalysis in patients under 3 and 6 months, and the results were similar, except for the percentage of

Table 1 Characteristics of the episodes in the emergency department: RSV versus non-RSV bronchiolitis

\begin{tabular}{|c|c|c|c|c|}
\hline & $\operatorname{RSV}(n=157)$ & Non-RSV $(n=64)$ & OR $(95 \% \mathrm{CI})$ & $P$ value \\
\hline Age of $<3$ months, $\%$ & 49 & 33 & $1.97(1.07-3.62)$ & 0.029 \\
\hline Female gender, \% & 41 & 33 & $1.40(0.76-2.59)$ & 0.27 \\
\hline Duration of symptoms at presentation, mean $\pm \mathrm{SD}$, days & $2.78 \pm 2.06$ & $2.71 \pm 2.05$ & n.a. & 0.83 \\
\hline Respiratory rate higher than normal for age, $\%^{\mathrm{a}}$ & 76 & 80 & $0.79(0.39-1.62)$ & 0.53 \\
\hline Retractions moderate/severe, $\%$ & 69 & 73 & $0.82(0.42-1.57)$ & 0.55 \\
\hline Presence of wheeze, $\%$ & 39 & 44 & $0.83(0.46-1.51)$ & 0.59 \\
\hline Oxygen saturation at arrival in $\mathrm{ED}$, mean $\pm \mathrm{SD}, \%^{\mathrm{b}}$ & $94.92 \pm 4.47$ & $94.26 \pm 7.04$ & n.a. & 0.40 \\
\hline \multicolumn{5}{|l|}{ Presence of bacterial coinfections, $\%$} \\
\hline Sepsis & 1 & 0 & n.a. & n.a. \\
\hline Pneumonia associated & 9 & 3 & $3.03(0.67-13.75)$ & 0.15 \\
\hline Acute otitis media associated & 16 & 13 & $1.32(0.56-3.11)$ & 0.52 \\
\hline Urinary tract infection associated & 2 & 0 & n.a. & n.a. \\
\hline
\end{tabular}

$R S V$ respiratory syncytial virus, $E D$ emergency department, $O R$ odds ratio, $C I$ confidence interval, $S D$ standard deviation, n.a. not analyzed

${ }^{\mathrm{a}}$ Normal values for age groups were as follows: 0 to 1.9 months of age, 45 breaths per minute; 2 to 5.9 months of age, 43 breaths per minute; 6 to 11.9 months of age, 40 breaths per minute

${ }^{\mathrm{b}}$ In room air 
Table 2 Medications received among episodes of bronchiolitis: RSV versus non-RSV

\begin{tabular}{|c|c|c|c|c|}
\hline & $\begin{array}{l}\text { RSV } \\
(n=157)\end{array}$ & $\begin{array}{l}\text { Non- } \\
\text { RSV } \\
(n=64)\end{array}$ & $\begin{array}{l}\text { OR } \\
(95 \% \mathrm{CI})\end{array}$ & $P$ value \\
\hline \multicolumn{5}{|c|}{ Medication used during the past week, $\%$} \\
\hline $\begin{array}{l}\text { Inhaled } \beta \text {-receptor } \\
\text { agonist treatment }\end{array}$ & 24 & 34 & $0.59(0.31-1.10)$ & 0.10 \\
\hline Oral corticosteroids & 1.9 & 1.6 & $1.22(0.12-12.02)$ & 0.86 \\
\hline Antibiotics & 8 & 5 & $1.83(0.50-6.67)$ & 0.36 \\
\hline \multicolumn{5}{|c|}{ Medication received in the hospital, $\%$} \\
\hline $\begin{array}{l}\text { Inhaled } \beta \text {-receptor } \\
\text { agonist treatment }\end{array}$ & 28 & 47 & $0.44(0.24-0.80)$ & 0.008 \\
\hline Oral corticosteroids & 4 & 3 & $1.44(0.29-7.15)$ & 0.65 \\
\hline Antibiotics & 12 & 8 & $1.62(0.57-4.55)$ & 0.36 \\
\hline \multicolumn{5}{|c|}{ Medication received at discharge, $\%$} \\
\hline $\begin{array}{r}\text { Inhaled } \beta \text {-receptor } \\
\text { agonist treatment }\end{array}$ & 9 & 11 & $0.79(0.30-2.07)$ & 0.64 \\
\hline Oral corticosteroids & 0 & 0 & n.a. & n.a. \\
\hline Antibiotics & 16 & 13 & $1.32(0.56-3.11)$ & 0.52 \\
\hline
\end{tabular}

$R S V$ respiratory syncytial virus, $O R$ odds ratio, $C I$ confidence interval, n.a. not analyzed

patients who received inhaled $\beta$-receptor agonist treatment at the hospital in patients $<6$ months. Although in this age group $<6$ months, RSV episodes were also less likely to receive inhaled $\beta$-receptor agonist treatment at the hospital; the differences between groups were not significant (13 and $22 \%$ for RSV and non-RSV group, respectively, $P=0.18$ ).

Accuracy of the virologic testing for RSV in detecting outcomes in patients with bronchiolitis

Table 4 shows the sensitivity, specificity, and positive and negative likelihood ratios with the following $95 \% \mathrm{CI}$ of virologic testing for RSV in detecting at-risk patients for hospitalization, longer LOS, need and longer duration of oxygen therapy, and need of NGT. Among patients admitted at the ED with a diagnosis of bronchiolitis, the virologic testing for RSV had low specificity in detecting outcomes for all analyzed variables, and the positive LRs were slightly greater than 1 .

\section{Discussion}

In agreement with previous reports [6, 7], patients with RSV bronchiolitis were younger and male was the predominant gender. In this study, the groups did not differ with respect to the initial clinical presentation. Different from us, Bamberger et al. found that children with RSV had a more severe initial clinical presentation compared to those with non-RSV bronchiolitis [2].

Previous studies have identified that RSV patients were more likely to receive systemic corticosteroids [7] and that patients hospitalized with bronchiolitis were more likely to receive inhaled $\beta$-receptor agonist and antibiotics at the hospital [9]. In these studies, the authors suggested that clinicians used more treatments for sicker children, with the hope of some benefits, although recent recommendations and guidelines do not encourage this attitude as it does not shorten the natural course of bronchiolitis $[4,18]$.

Even though our study was not designed to investigate the effectiveness of specific treatments in patients with bronchiolitis, we found that children with RSV episodes were less likely to receive inhaled $\beta$ agonist at the hospital. However, interestingly, the groups did not differ with respect to the number of children who had received inhaled $\beta$-receptor agonist treatment before arriving at the ED and continued to

Table 3 Differences in clinical course and disease severity between RSV and non-RSV bronchiolitis

\begin{tabular}{|c|c|c|c|c|c|c|}
\hline & $\operatorname{RSV}(n=157)$ & Non-RSV $(n=64)$ & OR $(95 \% \mathrm{CI})$ & $P$ value & $\mathrm{aOR}(95 \% \mathrm{CI})$ & $P$ value \\
\hline Hospitalization in ward/ICU, \% & 83 & 67 & $2.46(1.26-4.81)$ & 0.008 & $2.17(1.09-4.32)$ & 0.03 \\
\hline Hospitalization in ICU, \% & 7.6 & 7.8 & $0.97(0.32-2.89)$ & 0.97 & n.a. & n.a. \\
\hline LOS of $>3$ days, $\%$ & 61 & 36 & $2.73(1.49-4.98)$ & 0.001 & $2.45(1.32-4.54)$ & 0.004 \\
\hline Oxygen therapy, $\%$ & 72 & 48 & $2.73(1.49-4.98)$ & 0.001 & $2.78(1.51-5.13)$ & 0.001 \\
\hline Duration of oxygen therapy of $>2$ days, $\%^{\mathrm{a}}$ & 60 & 29 & $3.69(1.55-8.74)$ & 0.003 & $3.15(1.30-7.62)$ & 0.01 \\
\hline Noninvasive ventilation, $\%$ & 7 & 5 & $1.53(0.41-5.68)$ & 0.52 & n.a. & n.a. \\
\hline Intubated, $\%$ & 0.6 & 0 & n.a. & n.a. & n.a. & n.a. \\
\hline Nasogastric tube, $\%$ & 44 & 23 & $2.56(1.32-4.94)$ & 0.005 & $2.14(1.07-4.29)$ & 0.03 \\
\hline Duration of NGT of $>3$ days, $\%{ }^{b}$ & 45 & 40 & $1.22(0.39-3.81)$ & 0.73 & n.a. & n.a. \\
\hline Reconsultation after ED discharge, \% & 12 & 8 & $1.62(0.57-4.55)$ & 0.36 & n.a. & n.a. \\
\hline
\end{tabular}

$R S V$ respiratory syncytial virus, $I C U$ intensive care unit, $E D$ emergency department, $L O S$ hospital length of stay, $N G T$ nasogastric tube, $O R$ odds ratio, $a O R$ odds ratio adjusted for age, $C I$ confidence interval, n.a. not analyzed

${ }^{a}$ Among 144 patients who needed oxygen therapy

${ }^{\mathrm{b}}$ Among 84 patients who needed a nasogastric tube 
Table 4 Sensitivity, specificity, and likelihood ratios of virologic testing for RSV in detecting at-risk patients for more severe disease $(n=221)$

\begin{tabular}{lllll}
\hline & $\begin{array}{l}\text { Sensitivity }(95 \% \text { CI }) \\
\text { [range] }\end{array}$ & $\begin{array}{l}\text { Specificity }(95 \% \text { CI) } \\
\text { [range] }\end{array}$ & $\begin{array}{l}\text { Positive LR (95 \% CI) } \\
\text { [range] }\end{array}$ & $\begin{array}{l}\text { Negative LR (95 \% CI) } \\
\text { [range] }\end{array}$ \\
\hline Hospitalization & $75(68-81)$ & $44(31-59)$ & $1.4(1.0-1.8)$ & $0.5(0.4-0.8)$ \\
LOS of $>3$ days & $80(72-87)$ & $40(31-49)$ & $1.3(1.1-1.6)$ & $0.5(0.3-0.7)$ \\
Needed oxygen therapy & $78(71-84)$ & $42(32-54)$ & $1.4(1.1-1.7)$ & $0.5(0.3-0.7)$ \\
Duration of oxygen therapy of $>2$ days & $88(79-93)$ & $33(23-45)$ & $1.3(1.1-1.6)$ & $0.4(0.2-0.7)$ \\
Needed nasogastric tube & $82(72-89)$ & $35(28-44)$ & $1.3(1.1-1.5)$ & $0.5(0.3-0.8)$ \\
\hline
\end{tabular}

$R S V$ respiratory syncytial virus, $L R$ likelihood ratio, $L O S$ hospital length of stay, $C I$ confidence interval

receive it in the hospital. The result of the RSV test was not known by the clinicians when treatment decisions were taken in the ED. Therefore, we assume that the management in the ED was not modified due to viral etiology. Furthermore, both groups showed no difference in initial clinical presentation at the ED; thus, we believe that disease severity also did not influence doctors' decision in ED. Unfortunately, it was not possible to evaluate whether previous knowledge of virus etiology influenced management decisions in the ward or whether the higher rates of inhaled $\beta$-receptor agonist in the non-RSV group were due to a better response in this group.

Even though we performed less exams compared to other studies, it is still important to question whether we are performing more radiographs than necessary as this may increase the amount of abnormal radiographic findings and the unnecessary use of antibiotics. In previous studies, where more chest radiographs were performed ( $88 \%$ of patients), a significantly higher proportion of focal opacities and atelectasis in the RSV group was found [6, 7]. In a randomized trial, children with suspected lower respiratory tract infection who underwent chest radiographs received more antibiotics without any difference in time until recovery [17]. It is also reported that about $25 \%$ of hospitalized children with bronchiolitis will have radiographic evidence of atelectasis or infiltrates, often misinterpreted as possible bacterial infection [1].

Our findings are consistent with those of previous studies that found that RSV patients had longer $\operatorname{LOS}[2,6,7,10,11]$ and were more likely to have more supplemental oxygen requirements $[6,7]$ and inadequate oral intake $[2,7,10]$. However, these studies did not evaluate the duration of NGT, and only one reported a significantly longer duration of oxygen therapy in RSV patients compared to nonRSV [11].

In our study, we aimed to evaluate the accuracy of virologic testing for RSV in detecting patients at risk for a more severe disease. We found that even though the group of children admitted with RSV bronchiolitis had a more severe clinical course, virologic testing at an individual level did not have good accuracy in identifying patients at risk for hospitalization, longer LOS, and higher oxygen and NGT requirements, certainly because there are many other variables involved in disease severity and not only the viral etiology. While statistically significant, the positive LRs that we found were only slightly greater than 1 . The low specificity values showed that we could increase the costs by performing viral tests only for prognosis proposal in about $60 \%$ of patients who would not have a prolonged stay and would not even be hospitalized.

Our study has potential limitations; it has a retrospective design, and we limited the identification of patients in the ICD, which may have underestimated the number of patients with bronchiolitis. We also included patients with more than one episode of bronchiolitis, and even though some investigators emphasize the importance of looking at bronchiolitis in only those without prior wheeze illness, this is not a consensus and heterogeneity in the population exists [18]. Our sampling of patients probably presents a greater disease severity as we included only the patients in which viral tests had been performed. Indeed, in our routine hospital protocol, the majority of tests are performed in hospitalized patients for room isolation procedure. This also explains the high rates of hospitalization we had in this group. However, the same type of bias can be applied to both groups, which makes the data set comparable; moreover, our main outcomes were factors that can be evaluated mainly in hospitalized patients.

Following bronchiolitis guidelines, saying that virologic testing for RSV obtained during peak RSV season demonstrates a high predictive value for the diagnosis [1], the viral diagnostic tests were performed mainly during the RSV season. For this reason, the detection of RSV was not uniform during the 2 years of retrospective study. However, in the months when RSV tests were not routinely performed, the incidence of all bronchiolitis episodes was low, which suggests that we did not lose many cases of RSV episodes due to lack of sample collection in the off-season periods (see Fig. 1). Besides, a very small proportion of non-RSV cases may also represent RSV infections that were not detected due to a falsenegative assay result. Another limitation is that we did not analyze other viruses and the possible coinfections; therefore, categorizing children only by the RSV status alone may have obfuscated real associations which are described in some studies [11]. 
In conclusion, etiologic diagnosis is mainly useful for cohorting patients and for infection control especially in hospitalized patients. RSV-positive patients have a heavier economical impact; indeed, our study and other studies have shown that these patients have generally longer LOS and higher oxygen therapy and NGT requirements $[2,6,7,10$, 11]. However, different from other studies, our study indicates that at an individual level, as a performance test, virologic testing alone seems insufficient to precisely predict the outcomes. Therefore, virologic testing should not be routinely done in all patients presenting at the ED because it does not predict outcomes or help in management decisions as it may increase costs without adding further information. These findings would benefit from additional validation and analysis in prospective studies.

Acknowledgements We would like to thank the Platform of Clinical Research of Children's Hospital, University Hospitals of Geneva, Switzerland.

Funding The completion of this project did not require background extern.

Conflict of interest The authors declare that they have no conflict of interest.

\section{References}

1. American Academy of Pediatrics (2006) Diagnosis and management of bronchiolitis. Pediatrics 118(4): 1774-1793

2. Bamberger E, Srugo I, Abu Raya B, Segal E, Chaim B, Kassis I, Kugelman A, Miron D (2012) What is the clinical relevance of respiratory syncytial virus bronchiolitis?: findings from a multicenter, prospective study. Eur J Clin Microbiol Infect Dis 31(12): 3323-3330

3. Barben J, Hammer J (2003) Current management of acute bronchiolitis in Switzerland. Swiss Med Wkly 133(1-2):9-15

4. Barben J, Kuehni CE, Trachsel D, Hammer J, Swiss Paediatric Respiratory Research (2008) Management of acute bronchiolitis: can evidence based guidelines alter clinical practice? Thorax 63(12):1103-1109

5. Damore D, Mansbach JM, Clark S, Ramundo M, Camargo CA Jr (2008) Prospective multicenter bronchiolitis study: predicting intensive care unit admissions. Acad Emerg Med 15(10):887-894
6. Garcia CG, Bhore R, Soriano-Fallas A, Trost M, Chason R, Ramilo O, Mejias A (2010) Risk factors in children hospitalized with RSV bronchiolitis versus non-RSV bronchiolitis. Pediatrics 126(6): e1453-e1460

7. Hervas D, Reina J, Yanez A, del Valle JM, Figuerola J, Hervas JA (2012) Epidemiology of hospitalization for acute bronchiolitis in children: differences between RSV and non-RSV bronchiolitis. Eur J Clin Microbiol Infect Dis 31(8):1975-1981

8. Manoha C, Espinosa S, Aho SL, Huet F, Pothier P (2007) Epidemiological and clinical features of hMPV, RSV and RVs infections in young children. J Clin Virol 38(3):221-226

9. Mansbach JM, Clark S, Christopher NC, LoVecchio F, Kunz S, Acholonu U, Camargo CA Jr (2008) Prospective multicenter study of bronchiolitis: predicting safe discharges from the emergency department. Pediatrics 121(4):680-688

10. Mansbach JM, Piedra PA, Teach SJ, Sullivan AF, Forgey T, Clark S, Espinola JA, Camargo CA Jr (2012) Prospective multicenter study of viral etiology and hospital length of stay in children with severe bronchiolitis. Arch Pediatr Adolesc Med 166(8):700-706

11. Marguet C, Lubrano M, Gueudin M, Le Roux P, Deschildre A, Forget C, Couderc L, Siret D, Donnou MD, Bubenheim M, Vabret A, Freymuth F (2009) In very young infants severity of acute bronchiolitis depends on carried viruses. PLoS ONE 4(2):e4596

12. Nair H, Nokes DJ, Gessner BD, Dherani M, Madhi SA, Singleton RJ, O'Brien KL, Roca A, Wright PF, Bruce N, Chandran A, Theodoratou E, Sutanto A, Sedyaningsih ER, Ngama M, Munywoki PK, Kartasasmita C, Simões EAF, Rudan I, Weber MW, Campbell H (2010) Global burden of acute lower respiratory infections due to respiratory syncytial virus in young children: a systematic review and meta-analysis. Lancet 375(9725):1545-1555

13. Norwood A, Mansbach JM, Clark S, Waseem M, Camargo CA Jr (2010) Prospective multicenter study of bronchiolitis: predictors of an unscheduled visit after discharge from the emergency department. Acad Emerg Med 17(4):376-382

14. Papoff P, Moretti C, Cangiano G, Bonci E, Roggini M, Pierangeli A, Scagnolari C, Antonelli G, Midulla F (2011) Incidence and predisposing factors for severe disease in previously healthy term infants experiencing their first episode of bronchiolitis. Acta Paediatr 100(7): e17-e23

15. Richard N, Komurian-Pradel F, Javouhey E, Perret M, Rajoharison A, Bagnaud A, Vernet G, Lina B, Floret D, Paranhos-Baccala G (2008) The impact of dual viral infection in infants admitted to a pediatric intensive care unit associated with severe bronchiolitis. Pediatr Infect Dis J 27(3):213-217

16. Sommer C, Resch B, Simoes EA (2011) Risk factors for severe respiratory syncytial virus lower respiratory tract infection. Open Microbiol J 5:144-154

17. Swingler GH, Hussey GD, Zwarenstein M (1998) Randomised controlled trial of clinical outcome after chest radiograph in ambulatory acute lower-respiratory infection in children. Lancet 351(9100):404-408

18. Zorc JJ, Hall CB (2010) Bronchiolitis: recent evidence on diagnosis and management. Pediatrics 125(2):342-349 